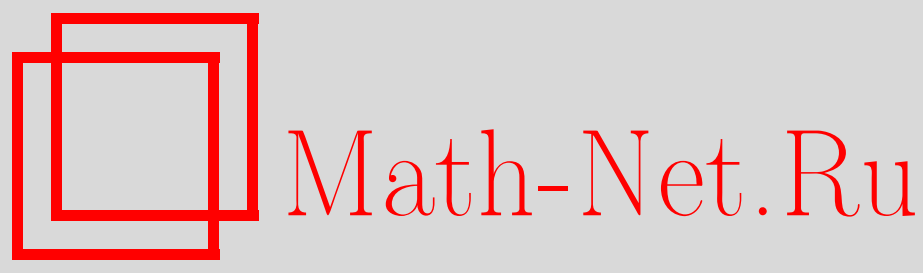

Р. В. Горбачев, Новое решение уравнения движения полевой теории суперструн, ТМФ, 2010, том 162, номер 1, 106-111

DOI: https://doi.org/10.4213/tmf6458

Использование Общероссийского математического портала Math-Net.Ru подразумевает, что вы прочитали и согласны с пользовательским соглашением http://www . mathnet.ru/rus/agreement

Параметры загрузки:

IP : 34.227 .88 .159

26 апреля 2023 г., 15:31:18

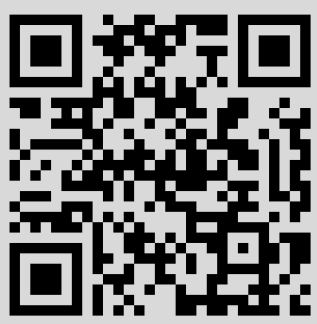




\section{НОВОЕ РЕШЕНИЕ УРАВНЕНИЯ ДВИЖЕНИЯ ПОЛЕВОЙ ТЕОРИИ СУПЕРСТРУН}

Построено новое решение уравнения движения в полевой теории суперструн. Показано, что это решение удовлетворяет двум гипотезам Сена и не требует "фантомных слагаемых".

Ключевые слова: струнная теория поля, D-браны, конденсация тахиона.

Посвящается памяти П. Б. Медведева

\section{1. ВВЕДЕНИЕ}

Струнная теория поля получила новое развитие после нахождения решения уравнения движения бозонной струнной теории поля [1]. Решение описывает вакуум, в котором нет возбуждений открытой струны и в котором распадаются D-браны. Первое аналитическое решение полевого уравнения движения суперструны [2], [3] было получено Эрлером [4]. Это решение имеет вид регуляризованной суммы

$$
\Phi=\lim _{N \rightarrow \infty}\left(\sum_{n=0}^{N} \varphi_{n}^{\prime}-\varphi_{N}-\frac{1}{2} \varphi_{N}^{\prime}\right)+\Gamma,
$$

где $\varphi_{n}=F c B \Omega^{n} c F, \Gamma=F B \gamma^{2} F$. Слагаемые $\varphi_{N}$ и $\varphi_{N}^{\prime}$ называются "фантомными слагаемыми". Эти слагаемые были добавлены для того, чтобы удовлетворить уравнению движения в "сильном" $\langle\langle\Phi, Q \Phi+\Phi \star \Phi\rangle\rangle=0[4]$ и "слабом" $\left\langle\left\langle\varphi_{m}, Q \Phi+\Phi \star \Phi\right\rangle\right\rangle=0$ [5] смысле. Как следствие мы можем доказать гипотезы Сена [4]

$$
E=-S(\Phi)=-\frac{1}{2}\langle\langle\Phi, Q \Phi\rangle\rangle-\frac{1}{3}\langle\langle\Phi, \Phi \star \Phi\rangle\rangle=-\frac{1}{2 \pi^{2}}
$$

* Российский университет дружбы народов, Москва, Россия.

E-mail: rgorbachev@mi.ras.ru 


\section{2. РЕШЕНИЕ}

В этой статье мы используем обозначения из работ [4], [6], [7] без детальных объяснений. Для того чтобы построить решение полевого уравнения движения суперструны, мы будем использовать следующие четыре струнных поля [4]:

$$
\begin{aligned}
K & =\text { грассманово четное поле }, & & \operatorname{gh} \#=0, \\
B & =\text { грассманово нечетное поле }, & & \operatorname{gh} \#=-1, \\
c & =\text { грассманово нечетное поле }, & & \operatorname{gh} \#=1, \\
\gamma^{2} & =\text { грассманово четное поле }, & & \operatorname{gh} \#=2,
\end{aligned}
$$

где gh\# - духовое число. Эти поля удовлетворяют следующим алгебраическим соотношениям:

$$
[B, K]=0, \quad[B, c]=1, \quad B^{2}=c^{2}=0, \quad\left[B, \gamma^{2}\right]=0, \quad\left[c, \gamma^{2}\right]=0,
$$

где $[\cdot, \cdot \cdot]$ обозначает градуированный коммутатор, и при действии БРСТ-оператора имеют вид

$$
Q c=c K c+\gamma^{2}, \quad Q B=K, \quad Q \gamma^{2}=c K \gamma^{2}-\gamma^{2} K c, \quad Q K=0 .
$$

Также мы определим $S L(2, \mathbb{R})$-инвариантный вакуум $\Omega=e^{K}$ и $F=\Omega^{1 / 2}$. Все произведения выше мы понимаем как струнное умножение.

Представление для полей $K, B, c, \gamma^{2}$ можно записать следующим образом:

$$
K=-\frac{\pi}{2}\left(K_{1}\right)_{L}|I\rangle, \quad B=-\frac{\pi}{2}\left(B_{1}\right)_{L}|I\rangle, \quad c=-\frac{1}{\pi} c(1)|I\rangle, \quad \gamma^{2}=\frac{1}{\pi} \gamma^{2}(1)|I\rangle,
$$

здесь $|I\rangle$ является единичным струнным полем, индекс $L$ обозначает интегрирование по положительной части единичного круга в направлении против часовой стрелки от $-i$ до $+i, K_{1}=L_{-1}+L_{1}, B_{1}=b_{1}+b_{-1}$ и $\gamma^{2}=\eta \partial \eta e^{2 \phi}$.

Теперь мы можем написать решение уравнения движения для суперструны

$$
Q \Phi+\Phi \star \Phi=0
$$

в виде

$$
\Phi=\left(-c-B \gamma^{2}+c K B c\right) \frac{1}{1-K} .
$$

Используя параметризацию Швингера [8], мы можем переписать $1 /(1-K)$ в виде

$$
\frac{1}{1-K}=\int_{0}^{\infty} d t e^{-t(1-k)}=\int_{0}^{\infty} d t e^{-t} \Omega^{t} .
$$

С учетом (4) и (5) легко проверить, что $\Phi$ удовлетворяет уравнению (6).

Как и в случае бозонного решения [8], мы можем получить вещественное решение суперструнного уравнения движения, используя калибровочное преобразование ${ }^{1)}$

$$
\widehat{\Phi}=U^{-1}(Q+\Psi) U=\frac{1}{\sqrt{1-K}}(Q+\Phi) \sqrt{1-K} .
$$

1) Условие вещественности означает, что $\Phi^{\ddagger}=\Phi$. Сопряжение $\ddagger$ определено как композиция эрмитова сопряжения и сопряжения Белавина-Полякова-Замолодчикова (БПЗ) [9]. 
Вещественное решение выглядит следующим образом:

$$
\widehat{\Phi}=\frac{1}{\sqrt{1-K}}\left(-c-B \gamma^{2}+c K B c\right) \frac{1}{\sqrt{1-K}},
$$

где

$$
\frac{1}{\sqrt{1-K}}=\frac{1}{\sqrt{\pi}} \int_{0}^{\infty} d t \frac{1}{\sqrt{t}} e^{-t} \Omega^{t}
$$

\section{3. КАЛИБРОВКА}

Решение Эрлера удовлетворяет калибровке Шнабла $\mathcal{B}_{0} \Phi=0$. Новое решение суперструнного уравнения движения удовлетворяет калибровке Эрлера-Шнабла [8]

$$
\mathcal{B}_{1, \frac{1}{1-K}} \Phi=0
$$

где $\mathcal{B}_{0}^{-}=\mathcal{B}_{0}-\mathcal{B}_{0}^{*}$ и

$$
\mathcal{B}_{f, g} \Phi=\frac{1}{2} f\left[\mathcal{B}_{0}^{-}\left(f^{-1} \Phi g^{-1}\right)\right] g,
$$

индекс * обозначает сопряжение БПЗ. Для того чтобы увидеть это, мы используем следующие тождества:

$$
\begin{aligned}
\frac{1}{2} \mathcal{B}_{0}^{-} K & =B, & \frac{1}{2} \mathcal{B}_{0}^{-} B & =0, \\
\frac{1}{2} \mathcal{B}_{0}^{-} c & =0, & \frac{1}{2} \mathcal{B}_{0}^{-} \gamma^{2} & =0,
\end{aligned}
$$

а также тот факт, что $\mathcal{B}_{0}^{-}$удовлетворяет правилу Лейбница. Калибровка Шнабла является частным случаем более общей калибровки Эрлера-Шнабла $\mathcal{B}_{0}=\mathcal{B}_{F, F}$.

\section{4. ДЕЙСТВИЕ}

В работе [8] было показано, что существует калибровочное преобразование между решением Шнабла и новым решением Эрлера-Шнабла. В этом разделе мы рассмотрим калибровочное преобразование между решением Эрлера полевого уравнения движения для суперструны и нашим новым решением. Для этого перепишем решения в одинаковой форме:

$$
\begin{aligned}
\Phi & =f c \frac{K B}{1-f g} c g-f B \gamma^{2} g \\
\Phi^{\prime} & =f^{\prime} c \frac{K B}{1-f^{\prime} g^{\prime}} c g^{\prime}-f^{\prime} B \gamma^{2} g^{\prime}
\end{aligned}
$$

где

$$
\begin{aligned}
& f^{\prime}=e^{K / 2}, \quad g^{\prime}=e^{K / 2} \quad \text { для решения Эрлера, } \\
& f=1, \quad g=\frac{1}{1-K} \quad \text { для нашего решения. }
\end{aligned}
$$

Используя (5), легко видеть, что эти решения калибровочно эквивалентны:

$$
\Phi^{\prime}=U^{-1}(\Phi+Q) U
$$



где

$$
\begin{aligned}
U & =1-f B c g+M f^{\prime} B c g^{\prime}, \\
U^{-1} & =1-f^{\prime} B c g^{\prime}+M^{-1} f B c g .
\end{aligned}
$$

Функция $M$ определена в виде

$$
M=\frac{1-f g}{1-f^{\prime} g^{\prime}}
$$

Заметим, что мы имеем такое же калибровочное поле $U$, как и в бозонной струне.

Следовательно, действие для нового решения равно (в предположении, что уравнение движения выполняется)

$$
S=\frac{1}{6}\left\langle\left\langle\Phi^{\prime}, Q \Phi^{\prime}\right\rangle\right\rangle=\frac{1}{6}\langle\langle\Phi, Q \Phi\rangle\rangle=\frac{1}{2 \pi^{2}}
$$

Как следствие энергия равна

$$
E=-S(\Phi)=-\frac{1}{2 \pi^{2}}
$$

Таким образом, новое решение удовлетворяет первой гипотезе Сена.

Вычислим непосредственно само действие. Для того чтобы корреляторы были ненулевыми, они должны иметь $\phi$-импульс, равный -2 . Так как оператор смены картины имеет $\phi$-импульс, равный -4 , только два слагаемых дадут нетривиальный вклад в действие:

$$
S=\frac{1}{6}\langle\langle\Phi, Q \Phi\rangle\rangle=\left\langle\left\langle c \gamma^{2} \frac{1}{1-K}\right\rangle\right\rangle+2\left\langle\left\langle B c K c \frac{1}{1-K} \gamma^{2} \frac{1}{1-K}\right\rangle\right\rangle .
$$

Для первого коррелятора мы имеем

$$
\begin{aligned}
\left\langle\left\langle c \gamma^{2} \frac{1}{1-K}\right\rangle\right\rangle & =\int_{0}^{\infty} d t e^{-t}\left\langle\left\langle c \gamma^{2} \Omega^{t}\right\rangle\right\rangle=\int_{0}^{\infty} d t e^{-t}\left\langle Y_{-2} c \gamma^{2}(0)\right\rangle= \\
& =\frac{1}{2 \pi^{2}} \int_{0}^{\infty} d t e^{-t} t^{2}=\frac{1}{\pi^{2}}
\end{aligned}
$$

для второго коррелятора

$$
\begin{aligned}
\left\langle\left\langle B c K c \frac{1}{1-K} \gamma^{2} \frac{1}{1-K}\right\rangle\right\rangle & =\left.\int_{0}^{\infty} d t d s e^{-t-s} \frac{d}{d n}\left\langle\left\langle\Omega^{s} B c \Omega^{n} c \Omega^{t} \gamma^{2}\right\rangle\right\rangle\right|_{n=0}= \\
& =\left.\int_{0}^{\infty} d t d s e^{-t-s} \frac{d}{d n} \frac{s+n+t}{2 \pi^{2}} n\right|_{n=0}=\frac{1}{\pi^{2}}
\end{aligned}
$$

Таким образом,

$$
S=\frac{1}{6}\left(\frac{1}{\pi^{2}}+\frac{2}{\pi^{2}}\right)=\frac{1}{2 \pi^{2}}
$$




\section{5. КОГОМОЛОГИЯ}

Следуя работам [7], [10], [11], непосредственно получаем доказательство отсутствия физических возбуждений в окрестности тахионного вакуума, или, что аналогично, отсутствие когомологии у оператора $Q \Phi$. Для этого необходимо найти оператор гомотопии $A$, удовлетворяющий выражению

$$
Q_{\Phi} A=Q A+[\Phi, A]=1
$$

Найдем оператор $A$ следующим образом [7]. Так как $A$ имеет духовое число -1 , предположим, что оно имеет вид

$$
A=B G(K)
$$

где $G$ - некоторое поле, зависящее только от $K$. Вычислим

$$
Q_{\Phi} A=K G-\left(c(1-K) B c+B \gamma^{2}\right) \frac{1}{1-K} B G-G B\left(c(1-K) B c+B \gamma^{2}\right) \frac{1}{1-K} .
$$

Если мы хотим сокращения $B$ и $c$, нам следует определить поле $G$ в виде

$$
G=-\frac{1}{1-K}
$$

Следовательно, оператор гомотопии имеет вид ${ }^{2)}$

$$
A=-B \frac{1}{1-K}=-B \int_{0}^{\infty} d t e^{-t} \Omega^{t} .
$$

\section{6. ЗАКЛЮЧЕНИЕ}

Мы представили простое решение уравнения движения для полевой теории суперструны. Это решение калибровочно эквивалентно решению Эрлера, а следовательно, действие для этого решения равно $1 / 2 \pi^{2}$. Также мы доказали, что оператор $Q_{\Phi}$ не имеет когомологий. Эта форма решения является предпочтительной, так как новая форма, как и в случае решения Эрлера-Шнабла, не требует регуляризации и "фантомных слагаемых". Было бы интересно найти простое аналитическое решение уравнений движения в полевой теории фермионной струны [12].

Благодарности. Автор благодарен И.Я. Арефьевой за полезные дискуссии. Работа частично поддержана РФФИ (гранты № 08-01-00798, 09-01-12179-офи), Программой поддержки ведущих научных школ (грант НШ-795.2008.1) и Федеральным агентством по науке и инновациям (на выполнение НИР № 02.740.11.5057).

\footnotetext{
2) Заметим, что оператор гомотопии для суперструны совпадает с оператором гомотопии для бозонной струны.
} 


\section{Список литературы}

[1] M. Schnabl, Adv. Theor. Math. Phys., 10:4 (2006), 433-501; arXiv: hep-th/0511286.

[2] I. Ya. Aref'eva, P. B. Medvedev, A. P. Zubarev, Nucl. Phys. B, 341:2 (1990), 464-498; Phys. Lett. B, 240:3-4 (1990), 356-362.

[3] C. R. Preitschopf, C. B. Thorn, S. A. Yost, Nucl. Phys. B, 337:2 (1990), 363-433.

[4] T. Erler, JHEP, 01 (2008), 013; arXiv: 0707.4591.

[5] I. Y. Aref'eva, R. V. Gorbachev, D. A. Grigoryev, P. N. Khromov, M. V. Maltsev, P. B. Medvedev, JHEP, 05 (2009), 050; arXiv: 0901.4533.

[6] Y. Okawa, JHEP, 04 (2006), 055; arXiv: hep-th/0603159.

[7] T. Erler, JHEP, 05 (2007), 083; arXiv: hep-th/0611200; JHEP, 05 (2007), 084; arXiv: hep-th/0612050.

[8] T. Erler, M. Schnabl, JHEP, 10 (2009), 066; arXiv: 0906.0979.

[9] M. R. Gaberdiel, B. Zwiebach, Nucl. Phys. B, 505:3 (1997), 569-624; arXiv: hep-th/9705038.

[10] I. Ellwood, B. Feng, Y.-H. He, N. Moeller, JHEP, 07 (2001), 016; arXiv: hep-th/0105024.

[11] I. Ellwood, M. Schnabl, JHEP, 02 (2007), 096; arXiv: hep-th/0606142.

[12] И. Я. Арефьева, Р. В. Горбачев, П. Б. Медведев, ТМФ, 158:3 (2009), 378-390; arXiv: 0804.2017. 